\title{
L'ingénierie sociale et territoriale dans les directions interministérielles de la cohésion sociale
}

Vers une co-construction des politiques publiques entre cadres d'État et habitants

\section{Ludovic Falaix}

\section{(2) OpenEdition}

\section{Journals}

Édition électronique

URL : http://journals.openedition.org/cdg/1839

DOI : $10.4000 /$ cdg. 1839

ISSN : 2107-7266

Éditeur

UMR 245 - CESSMA

Référence électronique

Ludovic Falaix, «L'ingénierie sociale et territoriale dans les directions interministérielles de la cohésion sociale », Carnets de géographes [En ligne], 1 | 2010, mis en ligne le 01 octobre 2010, consulté le 07 mai 2019. URL : http://journals.openedition.org/cdg/1839; DOI : 10.4000/cdg.1839

\section{(c) (i) $\ominus$}

La revue Carnets de géographes est mise à disposition selon les termes de la Licence Creative Commons Attribution - Pas d'Utilisation Commerciale - Pas de Modification 4.0 International. 


\title{
L'INGENIERIE SOCIALE ET TERRITORIALE DANS LES DIRECTIONS INTERMINISTERIELLES DE LA COHESION SOCIALE
}

\author{
Vers une co-construction des politiques publiques entre cadres d'Etat et \\ habitants
}

Ludovic Falaix

ludovic.falaix@yahoo.fr Conseiller d'Education Populaire et de Jeunesse Détaché en qualité de Directeur Enfance-Jeunesse à la ville de Saintes Doctorant en Aménagement de l'Espace, Urbanisme, Laboratoire SET, UMR 5603 - Université de Pau et des Pays de l'Adour

\begin{abstract}
Résumé
Cet article présente ce que peut recouvrir l'ingénierie sociale et territoriale au-delà de la définition technocratique proposée par le groupe de travail interministériel piloté par l'Inspection Générale des Affaires Sanitaires et Sociales. A partir des apports de la phénoménologie, il examine les mécanismes d'appropriation de l'espace par les habitants. Cet article souligne le caractère ontologique $\mathrm{du}$ rapport des habitants au territoire. Il met en exergue le fait que les individus " habitent l'espace en poète " en convoquant leur "Arkhe-Pensée» pour élaborer leur territorialisation. Une nouvelle approche de la construction des politiques publiques se dessine. Celle-ci consiste à s'affranchir de diagnostics fondés sur des analyses quantitatives, dont l'échec des politiques urbaines pointe les limites, au bénéfice de la prise en compte des aspirations des habitants dans la construction des politiques publiques.
\end{abstract}

La réforme de l'Etat territorial impulsée lors du conseil de modernisation des politiques publiques du 11 juin 2008 engendre de profonds bouleversements dans l'organisation fonctionnelle des services publics d'Etat. En effet, l'objet de cette réforme est de rationaliser le fonctionnement, les compétences, l'efficience des administrations et des services déconcentrés afin de garantir un meilleur service public aux administrés et de lutter contre les disparités sociales et territoriales. Ainsi, les circulaires du Premier Ministre du 19 mars et du 17 juillet 2008 prévoient le regroupement des Affaires Sanitaires et Sociales, de la Jeunesse et des Sports et des services de l'Agence pour la Cohésion Sociale et l'Egalité des chances dans des Directions Interministérielles Régionales de la Jeunesse, des Sports et de la Cohésion Sociale (DRJSCS). A l'échelle départementale, de nouvelles directions interministérielles de la Cohésion Sociale (DDCS) et/ou de la Protection des Populations (DDCS et/ou PP) voient le jour. Au final, c'est une reconfiguration des services déconcentrés qui est effective depuis le premier janvier 2010. Le périmètre des missions qui incombent désormais aux agents des nouvelles directions interministérielles régionales ou 
départementales est sans ambigüité ${ }^{1}$. Il convient d'agir en faveur de la cohésion sociale afin de réduire les «fractures» qui seraient flagrantes à l'échelle hexagonale. Ces fractures affectent certaines catégories d'habitants et il parait enrichissant de prendre en compte leurs aspirations dans la construction des projets socio-territoriaux formalisés par les pouvoirs publics. En effet, à l'heure où l'action publique recommande davantage de démocratie participative, cet article tend à analyser dans quelles mesures la participation des habitants peut constituer une valeur ajoutée dans la définition des politiques publiques locales en faveur du développement socio-territorial. Il devient alors intéressant d'appréhender si les bouleversements structurels qu'impose la Révision Générale des Politiques Publiques (RGPP) impulsent ou freinent cette participation et cette co-construction.

Cette réflexion n'implique pas pour autant une stigmatisation des mutations qui s'amorcent ou une volonté de verser dans une quelconque nostalgie d'une pratique professionnelle spécifique à chacun des réseaux ${ }^{2}$. L'idée est de présenter comment l'ingénierie sociale et territoriale, dont il conviendra de décliner ce qu'elle recouvre, autorise la participation des habitants dans l'élaboration des projets socio-territoriaux. Par conséquent, c'est bien les manières dont les habitants élaborent leurs territorialisations qu'il convient d'appréhender au cœur de cet article et les incidences qu'elles peuvent avoir sur la mise en œuvre opérationnelle des politiques publiques à l'échelle locale.

Peut-être en va-t-il de l'organisation des directions interministérielles de la cohésion sociale qui doit "permettre de développer des modes d'intervention nouveaux, mieux adaptés à la diversité des situations à traiter, s'appuyant sur une expertise renforcée $e^{3} » ?$ Preuve surement, à travers cette déclaration du premier ministre, que, jusqu'ici, les politiques publiques souffraient d'un manque d'efficacité. Efficacité dans l'expertise que nous pensons devoir inscrire justement dans la participation et la co-construction avec les habitants concernés.

\section{L'exemple des violences urbaines ou l'échec de certaines formes de politiques publiques}

Les violences urbaines, entendues comme "l'ensemble des transgressions quotidiennes des règles symboliques ou morales érigées en normes » (Lévy et Lussault, 2003 : 995) semblent récurrentes en France depuis plusieurs décennies. Elles se caractérisent par des émeutes urbaines qui sont largement relayées dans les médias. Ces débordements paroxysmiques, qui interpellent les pouvoirs publics, donnent lieu à la création d'établissements publics chargés de la mise en œuvre de politiques spécifiques afin de les prévenir. Néanmoins, malgré les nombreuses mesures prises par les pouvoirs publics, force est de constater que les difficultés persistent.

\footnotetext{
${ }^{1}$ Pour obtenir la liste exhaustive des missions : consulter le décret $n^{\circ} 2009-1540$ du 10 décembre 2009 relatif à l'organisation et aux missions des directions régionales de la jeunesse, des sports et de la cohésion sociale, ainsi que le décret $n^{\circ} 2009-1484$ du 3 décembre 2009 relatif aux directions départementales interministérielles.

${ }^{2}$ A cet égard, les compétences techniques et pédagogiques des agents du secteur Jeunesse ont été forgées à l'aune de l'Education Populaire et recouvrent des postures professionnelles spécifiques fondées sur l'expertise, la formation et l'accompagnement. Voir :

- Jean-Marie Mignon. Une histoire de l'éducation populaire. Paris : La découverte, 2007. 258 p. Collection Alternatives Sociales.

- Michel Héluwaert. Les conditions d'apparition du ministère de la Jeunesse et des Sports. Revue du comité d'histoire des ministères chargés de la jeunesse et des sports. INJEP. Novembre 2002. pp. 25-37.

${ }^{3}$ Circulaire du Premier Ministre du 19 septembre 2008 intitulée : «Lancement de la phase de préfiguration des nouvelles Directions Régionales de la Jeunesse, des Sports et de la Cohésion Sociale (DRJSCS) ».
} 
La récurrence des violences urbaines serait-elles le reflet des mauvais diagnostics qui concourent à l'élaboration des mesures de prévention et pourtant ne permettent pas de gommer les disparités sociales et spatiales au sein des territoires comme cela semble avoir été le cas pour la politique de la ville ${ }^{4}$ ? Le cas échéant, comment réfléchir à une nouvelle approche afin que ces diagnostics socio-territoriaux, qui président à l'élaboration de mesures concrètes, puissent permettre aux pouvoirs publics et aux acteurs du développement des territoires d'apporter des réponses adaptées afin que les violences urbaines, entendues comme des manifestations de l'exaspération face aux inégalités sociales et territoriales, ne soient plus aussi fréquentes ? Cet aspect mérite d'être examiné dans la mesure où "l'élaboration d'une politique publique consiste d'abord à construire une représentation, une image de la réalité sur laquelle on veut intervenir. C'est en référence à cette image cognitive que les acteurs organisent leur perception du problème, confrontent leurs solutions et définissent leurs propositions d'action: on appellera cette vision du monde le référentiel d'une politique » (Muller [1990], $2003: 65$ ).

L'enjeu relève d'une acuité nouvelle à l'heure où la réorganisation des services de l'Etat doit permettre d'une part, l'inclusion sociale des personnes les plus démunies, la promotion du lien social, de l'autonomie des personnes et leur développement personnel et, d'autre part, un rééquilibrage des disparités territoriales. L'atteinte de ces objectifs nécessite donc un recentrage des interventions de l'Etat sur l'ingénierie sociale et territoriale afin de construire de nouveaux référentiels. L'accent doit être mis sur les territoires prioritaires en proie à de profondes difficultés. A cet égard, les Directions Régionales de la Jeunesse, des Sports et de la Cohésion Sociale (DRJSCS), ainsi que les Directions Départementales Interministérielles de la Cohésion Sociale (DDCS) sont chargées de promouvoir un large éventail de politiques publiques afin d'enrayer les profondes disparités sociales et territoriales. Néanmoins, ces entités administratives sont confrontées à des enjeux de fédération de réseaux qui recouvrent des métiers, des logiques d'action, des modes d'intervention et des cultures très différentes comme l'illustre la figure ci-dessous.

\footnotetext{
${ }^{4}$ Voir la position de Luc Bronner exprimée, à partir de données quantitatives, dans un article publié dans Le Monde du 23 Octobre 2009 au sujet de la politique pilotée par Fadela Amara.
} 


\section{Culture professionnelle des trois réseaux composant les Directions Régionales interministérielles de la Jeunesse, des Sports et de la Cohésion Sociale}

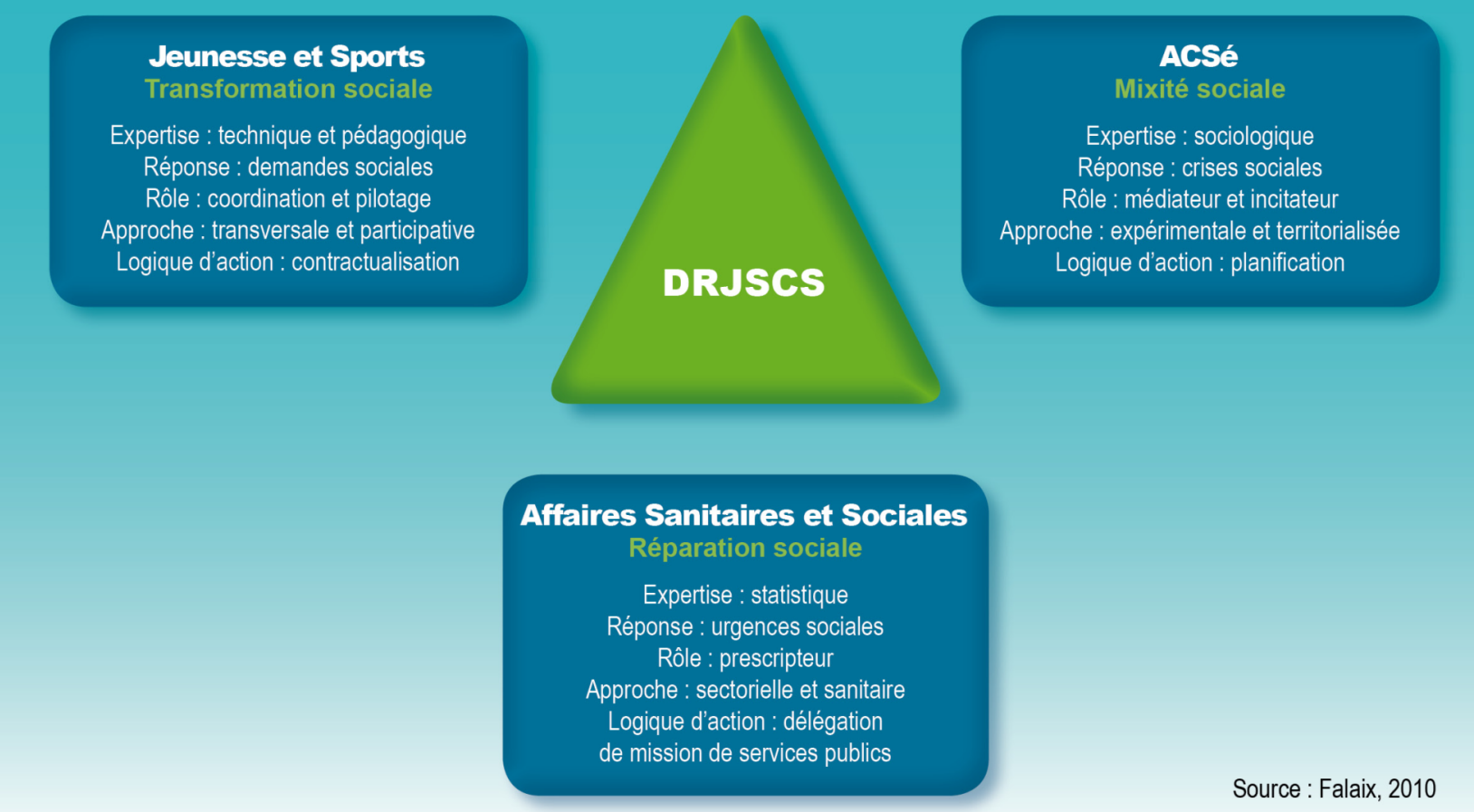

C'est donc un processus d'acculturation qui se dessine pour les agents appelés à exercer au sein des nouveaux services déconcentrés. Dans ce contexte, l'ingénierie sociale et territoriale peut apparaître comme un élément commun de posture et d'action pour différents corps de métiers qui doivent travailler ensemble dans les services déconcentrés de cohésion sociale. Les agents doivent donc construire d'autres représentations, débarrassées des stéréotypes et des caricatures, pour forger de nouvelles images sociales et territoriales des zones prioritaires afin d'élaborer de nouveaux référentiels dans le cadre des politiques publiques. C'est en ce sens que le concept d'ingénierie sociale et territoriale est mobilisé par les intellectuels chargés d'impulser la Révision Générale des Politiques Publiques.

\section{Révision Générale des Politiques Publiques et ingénierie sociale}

La RGPP entérine un bouleversement majeur quant aux postures professionnelles des agents d'Etat au bénéfice de la fonction d'ingénierie sociale et territoriale. Elle devient désormais le ressort paradigmatique sur lequel s'appuie la conduite des politiques publiques en matière de cohésion sociale ${ }^{5}$. Quelle que soit leur administration d'origine, les agents doivent donc s'en emparer pour garantir l'efficience des politiques publiques. En effet, la synthèse d'un groupe de travail sur l'ingénierie sociale, présidé par Annick Morel, Inspectrice Générale des Affaires Sanitaires et Sociales (IGASS), propose une définition du terme, présente les enjeux liés à l'application de ce concept dans le champ des politiques publiques, évoque les processus méthodologiques et les postures professionnelles requis ainsi que les incidences de son application dans le cadre de la réorganisation des services de l'Etat:

\footnotetext{
${ }^{5}$ La circulaire du Premier Ministre du 19 septembre 2008 relative à la création des DRJSCS emploie le terme d'ingénierie sociale pour désigner l'une des missions de ces nouvelles directions régionales dont la fonction d'appui et d'expertise est clairement identifiée.
} 
- " Définition : l'ingénierie sociale peut se définir comme une fonction d'ensemblier ou "d'assemblier» qui se situe dans la pratique, l'action, l'intervention, et apporte, ou aide à trouver, des solutions pour favoriser la résolution de problèmes dans un champ sociétal. L'ingénierie sociale comporte une fonction politique (elle doit tenir une vision des rapports sociaux et des rôles des institutions dans la structuration de ces rapports), technique et s'appuie sur des militants de l'action publique qui interviennent dans la perspective de l'intérêt général.

- Enjeux : la "prospérité » du concept est ainsi fortement reliée à l'émergence de la notion de gouvernance, définie comme un nouveau mode d'intervention de l'Etat, qui fait appel à la coopération des acteurs, à leur interaction pour la co-construction d'actions publiques. Autrement dit, l'Etat ne doit plus faire mais " faire-faire ».

- Processus méthodologiques et postures professionnelles: le titulaire d'une fonction d'ingénierie sociale ne tire pas sa légitimité de sa position dans une hiérarchie. L'ingénierie sociale trouve sa pleine force quand elle permet de réinterroger la commande dans le cadre d'une maîtrise d'œuvre intelligente prenant appui sur une connaissance des territoires et des contextes.

- Réorganisation de l'Etat, cohésion sociale et ingénierie sociale : l'Etat affirme ainsi sa légitimité dans son rôle de garant de la cohésion sociale pour deux raisons principales : la première est l'affirmation de la fonction de solidarité nationale, la seconde tient au fait que l'Etat est un fédérateur légitime, un animateur pour traiter le sujet de l'équité territoriale et sociale ».

Les agents d'Etat, issus des trois réseaux (Jeunesse et Sports, Agence de la Cohésion Sociale et de l'égalité des chances - ACSé, Affaires Sanitaires et Sociales) et aujourd'hui regroupés au sein d'un même service déconcentré interministériel de la Cohésion sociale, doivent réfléchir à la manière dont ils peuvent, au regard de leurs compétences en termes d'expertise, adopter une posture professionnelle qui leur permette de prendre place au cœur $\mathrm{du}$ processus de construction d'un nouveau référentiel, véritable moteur des politiques publiques. Néanmoins, à l'heure où le gouvernement demande à ses agents de "faire-faire ", quelles sont les références théoriques sur lesquelles ceux-ci peuvent prendre appui pour construire une posture cohérente et partagée en matière de développement social et territorial des zones prioritaires telles que définies dans les diverses instructions ministérielles?

\section{Pierre Muller et les politiques publiques}

Les travaux de Pierre Muller permettent aux agents d'Etat, regroupés dans les directions interministérielles de la cohésion sociale, appelés à "faire faire ", d'interroger leurs pratiques professionnelles et leurs compétences respectives en matière d'ingénierie sociale et territoriale pour tendre vers cet impératif gouvernemental. A l'aune des recommandations formulées par le chercheur, les agents d'Etat des directions interministérielles de la cohésion sociale peuvent être appelés à jouer un rôle nouveau dans la construction des politiques publiques. Ils pourraient en qualité de "médiateurs " véhiculer l'idée que la participation des populations locales est prépondérante dans le cadre de la 
construction de politiques publiques censées résoudre des problématiques dont les caractéristiques dépendent étroitement du contexte social et territorial local.

En effet, l'auteur présente le référentiel d'une politique comme "un ensemble de prescriptions qui donnent du sens à un programme politique en définissant des critères de choix et des modes de désignation des objectifs. Le référentiel se structure selon quatre niveaux:

- Les valeurs : sont les représentations fondamentales sur ce qui est bien ou mal. Elles définissent le cadre global de l'action publique,

- Les normes : définissent des écarts entre le réel perçu et le réel souhaité. Elles définissent des principes plus que des valeurs. A titre d'exemple : il faut promouvoir la cohésion sociale (le réel perçu est une société où se creusent les inégalités, le réel souhaité est que ces inégalités soient comblées),

- Les algorithmes : sont des relations causales qui expriment une théorie de l'action. Ils peuvent être exprimés sous la forme «si... alors». Par exemple: "Si nous accompagnons les publics les plus vulnérables alors nous réduirons les inégalités sociales et territoriales »,

- Les images: sont des vecteurs implicites de valeurs, de normes ou même d'algorithmes. Elles font sens immédiatement sans long détour discursif. Elles constituent donc un élément central du référentiel ».

D'autre part, l'auteur distingue le référentiel global et le référentiel sectoriel :

- "Le référentiel global: est une représentation générale autour de laquelle vont s'ordonner et se hiérarchiser les différentes représentations sectorielles. Il est constitué d'un ensemble de valeurs fondamentales qui constituent les croyances de base d'une société, ainsi que de normes qui permettent de choisir entre des conduites. Les valeurs et les normes qui caractérisent le référentiel global évoluent en fonction de l'histoire sociale.

- Le référentiel sectoriel : est une représentation du secteur, de la discipline ou de la profession. La configuration d'un secteur dépend étroitement de la représentation que l'on se fait de la place du secteur dans la société. A titre d'exemple, la jeunesse référentiel sectoriel - peut être appréhendée comme une ressource ou comme un problème » (Muller [1990], 2003 :57-68)

Selon Pierre Muller: "l'opération d'encastrement du référentiel sectoriel dans le référentiel global est l'acte fondateur d'une politique publique. Les acteurs qui réalisent cette opération de transaction sont les médiateurs d'une politique publique. Les médiateurs produisent donc du sens (prise de parole) et élaborent des stratégies (prise de pouvoir) afin de se positionner au sein d'un champ de pouvoir» (Muller [1990], 2003 :69)

Le rôle des médiateurs consiste donc à véhiculer l'idée que les préoccupations et aspirations des populations doivent être entendues dans le cadre de la construction des politiques publiques. Aux agents d'Etat des directions interministérielles de la cohésion 
sociale de présenter les discours des populations locales comme une ressource sociale et territoriale. Une autre gouvernance des politiques publiques, fondée sur la participation des habitants, émergerait. Cela dit comment encourager la participation sans appréhender les processus de socialisation et de territorialisation des habitants et décrypter les aspirations, en termes de besoins socio-territoriaux, qu'ils recouvrent?

\section{Crise sociale du territoire et posture intellectuelle}

Les territoires sont généralement appréhendés à travers le prisme de la pensée positiviste. C'est donc davantage les potentialités des territoires en termes de ressources économiques, environnementales, démographiques, numériques... qui sont examinées. Cependant très vite apparaissent les limites d'une telle approche. En effet, en s'affranchissant de la dimension existentielle du processus de territorialisation, ces analyses ne peuvent en témoigner. Ces approches consistent donc davantage à proposer des modèles théoriques qui rendent compte du fonctionnement des territoires. Elles ne nous permettent donc pas d'être renseignés sur l'acte premier qu'est " habiter ». En d'autres termes, nous ne sommes guère renseignés sur le projet téléologique des habitants, ni sur le fait de savoir si ce projet concoure à aller dans le même sens que ce pour quoi les acteurs de cette cohésion sociale construisent les politiques publiques. Or, nous avons pu examiner, à travers l'exemple des politiques de la ville, que le caractère normatif des projets d'actions formalisés par les représentants de l'Etat n’engendrait pas de réelles améliorations.

Comment dès lors ré-introduire l'espace habité comme une manifestation des expériences intuitives et conceptuelles que s'en construisent les sujets ? Ne convient-il pas alors de se représenter les habitants comme des êtres qui construisent leurs rapports à l'espace en fonction de la manière dont ils captent et traduisent les stimuli de leur environnement social ? Interpréter les manières dont les individus vivent leur rapport social et spatial au territoire est une opportunité pour les agents exerçant au cœur des directions interministérielles de véhiculer de nouveaux référentiels sectoriels.

Autrement dit, il convient de réfléchir à la dimension ontologique du phénomène territorial pour appréhender les modalités que convoquent les individus pour élaborer leur socialisation et leur territorialité. Il s'agit alors de rendre compte de la poïesis des acteurs, c'est-à-dire de leur potentiel d'action et de production en termes de socialisation et de territorialisation, afin de mettre en lumière la dimension affective des processus sociaux et spatiaux. Aussi, nous pourrons lire l'émergence des territoires, entendus comme espaces de lien social, comme l'expression d'une volonté «d'habiter l'espace en poète » (Heidegger [1954], $2001: 224-245)^{6}$.

Mais pour saisir le caractère ontologique qui préside à la construction d'un territoire, encore faut-il prendre en compte les aspirations individuelles et collectives dans le cadre de la construction des politiques publiques pensées comme leviers du développement socioterritorial.

Dans ce contexte, l'analyse socio-géographique constitue un outil d'analyse et de réflexion pour les acteurs du développement socio-territorial. En effet, traduire les aspirations

\footnotetext{
${ }^{6}$ Heidegger emprunte cette expression à Friedrich Hölderlin.
} 
collectives et individuelles en termes de désirs sociaux et d'appropriation spatiale permet aux acteurs institutionnels d'intégrer leurs exigences sociales. L'enjeu est de mettre en évidence le fait que, pour tous, l'existence heureuse n'est effective qu'à la condition "d'éprouver la dimension géo-poétique des lieux » (Ferrier, 1998). Or, n'est-ce pas la mission qui incombait jusqu'ici aux agents d'Etat qui, au contact du tissu associatif, des acteurs du monde sportif, culturel et socio-éducatif, s'employaient à relayer et accompagner leurs projets social et territorial?

Si l'espace urbain est en crise, c'est peut-être parce que "la ville ne fait plus société » (Donzelot, 1999). Dans ce contexte, l'hypothèse est la suivante: certains individus nourriraient le sentiment d'une déterritorialisation, c'est-à-dire qu'ils prendraient conscience de la généralisation d'un urbanisme imposant un rapport rationnel et pragmatique à l'espace qui ne leur permettrait plus d'expérimenter le caractère identitaire, relationnel et social de l'espace. En effet, la dynamique de normalisation, de hiérarchisation et de codification spatiale introduit la multiplication de "non-lieux » (Augé, $2002: 100$ ) c'est-à-dire des espaces qui n'ont plus de caractère identitaire et relationnel. Ainsi, plus la société se modernise, plus elle fragmente l'espace imposant alors des frontières sociales et spatiales hermétiques et symboliques. A présent, aux formes sociales et urbaines de la ville s'associent les termes de relégation, gentrification, ghettoïsation. (Donzelot, 2004). Malgré tout, force est de constater, qu'au cœur de certains territoires, s'amplifient les stratégies de l'entre-soi rompant avec le propre de la ville qui serait, à en croire Jacques Donzelot, de promouvoir la mixité sociospatiale. On assiste alors à des formes de repli communautaire, dont les gated communities sont l'exemple le plus abouti, qui exacerbent les logiques de cloisonnement social et spatial (Rifkin, 2000 : 151-162). Or, ce processus de séparation, de segmentation qui affecte la ville engendre de profondes frustrations et celles-ci nous invitent à formaliser de nouvelles grilles d'analyse pour les comprendre et construire des outils pour élaborer des politiques publiques efficaces.

\section{Habiter ou le processus de territorialisation}

Le territoire est "le morceau d'espace dans lequel un individu peut agir pour se gratifier " (Laborit [1974], $2000: 80$ ). Autrement dit, il est un "espace pratiqué 》 (De Certeau, 1990 :173). Plus encore il est un " espace consacré », c'est-à-dire un espace au sein duquel l'individu expérimente sa propre intimité, son rapport au monde et aux autres (Eliade [1965], 2002 :26). Or, à l'heure actuelle, quels territoires restent-ils aux individus, notamment les plus précarisés, afin qu'ils puissent agir pour se gratifier en s'appropriant les lieux, en consacrant les espaces, en les pratiquant sur un registre intuitif et émotionnel ? A ce propos, en 1997, n'est-ce pas ce que rapportaient les chanteurs du groupe de hip-hop marseillais IAM dans leur chanson, extraite de l'album " L'école du micro d'argent », intitulée "demain c'est loin ». Ne relayaient-ils pas, au nom d'une frange de la jeunesse des quartiers populaires, le mal-être quant à leur rapport à l'espace que celle-ci pouvait éprouver : "Regarde la rue, ce qui change? Y'a que les saisons, tu baves du béton, craches du béton, chies du béton... Les élus ressassent rénovation ça rassure, mais c'est toujours la même merde, derrière la dernière couche de peinture $»$.

Comprendre l'actuelle "révolution de la cosmologie » (Sloterdijk, 2003 :22), c'est-àdire la perte des lieux que produisent les habitants pour se sentir vivants et insérés dans le tissu social, c'est être en mesure d'interpréter, sinon de légitimer, certaines attitudes trop rapidement stigmatisées comme déviantes. Mais comprendre que les individus sont en proie à 
certaines formes de mal-être socio-territorial, c'est pouvoir agir afin d'y remédier. C'est pouvoir conceptualiser et modéliser des stratégies d'intervention pour mettre fin à cette crise de l'urbanité. C'est assurer la promotion d'événements sociaux et territoriaux qui permettent de prévenir ces manifestations de souffrance. C'est être capable d'anticiper ces souffrances par des politiques publiques de transformation sociale et territoriale. C'est ne plus seulement proposer des politiques publiques de réparation sociale et territoriale qui agissent sur les causes et non les effets des crises socio-territoriales. Or, accompagner, soutenir, encourager l'organisation de manifestations sportives, d'événements culturels et socio-éducatifs, entendus comme des temps majeurs de transformation sociale et territoriale, est le propre des missions des agents d'Etat exerçant dans les services déconcentrés de la cohésion sociale. C'est en ce sens qu'ils ont un rôle à jouer en qualité de médiateurs. Néanmoins, encore faut-il sensibiliser les cadres d'Etat sur les modalités que convoquent les individus pour construire leurs socialisations et leurs territorialisations afin qu'ils soient en mesure d'élaborer de nouvelles méthodologies de travail fondées sur le recueil des aspirations individuelles et collectives des habitants.

Dans cette perspective, l'approche dialogique et phénoménologique permet de révéler les actions gratifiantes et les rapports sociaux que les individus aspirent à vivre au cœur du territoire qu'ils habitent (Hoyaux, 2006 : 271-285). Il s'agit alors de privilégier la « relation de l'homme et de l'espace qui n'est rien d'autre que l'habitation pensée dans son être " (Heidegger [1954], 2001 :188). Autrement dit, il s'agit de mettre en exergue les manières dont les individus habitent leur espace. C'est avancer l'idée que les individus veulent faire de "l'espace avant tout un champ de valeurs, transposition de l'imaginaire dans le réel plus que le réel dans l'imaginaire » (Moles et Rohmer, 2003 :53).

La manière dont les individus habitent l'espace doit tenir une place prépondérante car "l'habitat est bien le terme constitutif du rapport sujet/lieu, rapport où la nature du lieu est fonction de celle du sujet" (De Radkowski, 2002 :24). Comprendre les relations qu'entretiennent les habitants avec le monde qui les entoure invite donc à saisir les manières dont ils appréhendent leur environnement socio-spatial. Adopter cette posture humaniste et philanthropique, c'est donc rompre avec une politique réductrice qui homogénéise les territoires en appliquant partout les mêmes recettes d'aménagement et de développement social, culturel, éducatif, sportif, économique, environnemental. C'est refuser l'hégémonie d'un référentiel global qui préside à l'élaboration des politiques publiques en s'affranchissant d'une expertise sectorielle.

\section{Ne plus aménager et développer le territoire " pour " les habitants mais " avec "}

Les acteurs du développement social et territorial s'emploient trop souvent à élaborer des diagnostics en fonction des composantes économiques, environnementales et sociales. Les rapports sont donc fondés sur l'analyse d'indicateurs quantitatifs et occulte le caractère qualitatif pourtant primordial quand il s'agit de traiter avec de $1^{1}$ humain ${ }^{7}$. Ainsi, rares sont les

\footnotetext{
7 A titre d'exemple, le dernier rapport 2009 publié par l'ONZUS contient de nombreux indicateurs qui permettent d'apprécier les résultats des politiques publiques en matière d'emploi, d'équipements, de santé, de sécurité publique. La parole des habitants concernés par la mise en œuvre de ces politiques publiques n'est pas retranscrite pour saisir leur perception de ces politiques. Volontairement provocateur, posons la question suivante : Comment peut-on être sûr que la construction d'un nouveau gymnase, d'une nouvelle MJC soient au cœur de leurs préoccupations et de leurs désirs en termes d'aménagements et d'équipements?
} 
projets de développement socio-territorial, construits à l'aune de ces rapports ou expertises quantitatives, qui accordent du crédit aux manières dont les individus souhaitent habiter leurs espaces. L'une des missions qui incombent aux agents des nouveaux services déconcentrés d'Etat serait donc de se positionner à l'interface entre les pouvoirs publics et les populations locales afin que les premiers prennent en considération les aspirations collectives des secondes. Dans le cadre de la politique de cohésion sociale, leur rôle serait donc de relayer le projet téléologique des habitants en privilégiant la concertation et le dialogue afin d'aider les habitants à élaborer, à fédérer, à formaliser et à élucider les manières dont ils envisagent les modalités d'un vivre ensemble. Or, c'est précisément le rôle des agents d'Etat lorsqu'ils participent à des comités de pilotage tels que ceux préconisés dans le cadre des Contrats Educatifs Locaux. A cet égard, ces perspectives ne s'inscrivent-elles pas en résonance avec les principes de l'urbanisme avancés par François Ascher, soit la nécessité de privilégier les objectifs par rapport aux moyens, d'adapter les projets urbains à la diversité des besoins et à la variété des demandes, de créer une nouvelle gouvernance urbaine... ? (Ascher, 2004 :79-99) Ces méthodes de travail qui privilégient la co-construction des missions de service public répondent aux préconisations présentées dans le rapport sur l'ingénierie sociale : "L'Etat ne doit plus faire mais faire-faire ».

\section{Convoquer l'Arkhé-Pensée}

Les individus convoquent leur pensée mythique pour vivre l'espace sur un registre où s'orchestrent leurs émotions, leurs désirs de s'éprouver, de se sentir exister... En effet, l'émergence de la territorialisation traduit une volonté de "vivre l'espace en poète ». Or ces espaces où l'individu exerce sa propre souveraineté, sont ceux de la nostalgie. Ces « espaces de la nostalgie " procèdent d'un "projet réel ou métaphorique d'échapper à l'espace et à la pensée trop unidimensionnelle de la civilisation contemporaine, pour revenir à des conditions qui ne dissocient pas la pensée mythique et la pensée rationnelle » (Roux, 1999 :49). Ces productions spatiales s'opèrent dans la mesure où la déterritorialisation est effective. Or, avec la normalisation des espaces, la déterritorialisation s'opère. Autrement dit, on assiste au passage d'une société où un espace restreint assure la transaction entre les éléments naturels, les êtres humains et les entités divines, et, représente en un point donné la totalité de l'univers, à une société où il devient exclusivement le support de l'interdépendance sociale.

Ainsi, plus la société se modernise, c'est-à-dire mobilise la raison au détriment de l'émotion, plus elle invite les individus à se projeter au cœur "d'espaces de la nostalgie ", véritable expression d'un déficit de territorialisation, dont les représentations et les usages sociaux s'appuient sur l'imaginaire des individus. La production de nostalgie s'organise donc selon un processus de rétroaction négative. Dès lors, cette expérience spatiale, qui participe d'un transfert des matrices sensitives des individus, répondrait du processus de déterritorialisation induit par la hiérarchisation, la codification, la standardisation de l'espace, véritables ressorts dogmatiques de la modernité. En effet, les individus, en conjuguant un nouveau rapport à l'espace construisent une logique visant à produire des lieux mythiques, des territoires sacrés que chaque individu s'approprie au titre de héros fondateur.

$\mathrm{Eu}$ égard à la rareté croissante des possibilités de revivre l'espace sur un plan mythique, on comprend pourquoi les individus se tournent de plus en plus vers ce type de productions spatiales qui leur permettent d'agir " pour se gratifier » et d'expérimenter du lien social. Ainsi, à titre d'exemple, l'essor des pratiques sportives urbaines, détournant les 
fonctionnalités du mobilier urbain, traduit cette volonté des individus de se re-territorialiser. Le graffiti répond également à cette logique : agir sur l'espace en y laissant la marque de son passage est une forme de territorialisation. De nouvelles stratégies territoriales s'affirment donc à partir de l'appréhension et de l'utilisation de nouveaux éléments de l'espace qui sont traduit par ces divers collectifs comme de précieuses ressources territoriales à protéger, à partager ou à défendre. Les individus mobilisent alors leur "Arkhe-Pensée » pour engager ce processus de territorialisation. En effet, l'Arkhe-Pensée telle que définie par Edgar Morin "correspond aux forces et formes originelles, principielles et fondamentales de l'activité cérébro-spirituelle. C'est une façon de concevoir la pensée symbolique-mythologique. L'Arkhe-Pensée se polarise sur la réalité subjective; mobilise une néo-pensée symboliquemythologique-magique... en ce sens où c'est la pensée rationnelle qui revitalise la pensée symbolique-mythologique-magique originelle. En effet, l'histoire contemporaine, tout en dissolvant les anciennes mythologies (pensée rationnelle), en secrète de nouvelles, et régénère de façon proprement moderne la pensée symbolique-mythologique-magique... Ainsi, l'objectivité et la subjectivité de la connaissance, relève non de deux compartiments distincts ou de deux sources différents, mais d'un circuit unique d'où elles vont se distinguer, puis éventuellement s'opposer, chacune nourrissant principalement l'une des deux pensées. Ce circuit unique est une boucle génératrice, l'Arkhe-Esprit, où se forme la représentation et le langage » (Morin [1986], 2001 :153-175).

Promouvoir ce type de rapport à l'espace, c'est-à-dire la possibilité pour les habitants d'expérimenter le caractère transcendantal de leurs rapports sociaux et territoriaux, c'est donc être en mesure de répondre aux aspirations collectives des citoyens. Là encore, nul ne doute que les agents d'Etat de la cohésion sociale ont toute légitimité pour assurer la promotion de ces opérations : raids sportifs urbains ${ }^{8}$, spectacles d'arts de la rue...

Par conséquent, il s'agit de décrypter à quels paradigmes ${ }^{9}$ répondent les productions de ces espaces poïétiques. Car, nous pouvons alors aborder l'espace physique comme le lieu où se projettent parfois les structures sociales; il constitue alors "l'une des médiations à travers lesquelles les structures sociales se convertissent en structures mentales et en systèmes de préférences " (Bourdieu, 1993 :249). L'intérêt d'une telle posture intellectuelle réside donc dans l'exploration des modes de lectures de l'espace et de l'environnement social, c'est-à-dire, l'exploration des images poïétiques, des imaginaires, des symboles, des mythes que mobilisent les individus afin de les transférer ${ }^{10}$ pour construire leur langage spatial, leur géographie existentielle, leur lien social. Or, d'une part, seule la proximité avec le terrain, les acteurs locaux, le tissu associatif, les habitants, et d'autre part, seuls le dialogue, l'échange, la

\footnotetext{
${ }^{8}$ A cet égard, Rama Yade a lancé les premiers Etats Généraux des Sports Urbains le 16 décembre 2009 au Stade Pierre de Coubertin à Paris. Lors de son allocution de clôture de ces Etats Généraux, la Secrétaire d'Etat aux Sports affirmait que: "Depuis des années, des décennies parfois, les sports urbains ont connu une forte croissance de leurs activités. Mais cette croissance s'est faite, c'est là le problème, à l'écart du monde sportif traditionnel et sans aucune reconnaissance des pouvoirs publics. Ce qui a entraîné avec le temps plusieurs difficultés, en particulier en matière d'accès des sports urbains à l'espace public, de sécurité des pratiques ou encore d'absence de formations adaptées ».

${ }^{9}$ Nous entendons par paradigme l'acception proposée par Edgar Morin, c'est-à-dire « un ensemble de concepts fondamentaux et de catégories maîtresses de l'intelligibilité articulés entre eux qui proposent des clefs d'interprétations du réel tout en restant implicites ». Edgar Morin. La méthode, 4 - Les Idées, leur habitat, leur vie, leurs mours, leur organisation. Paris : Seuil, 1991. p 218.

${ }^{10}$ Acception psychanalytique du terme, soit le processus par lequel le sujet reporte ses désirs, cette fois non sur l'analyste, mais sur l'espace. Freud S., [1917], 1993. Introduction à la psychanalyse. Paris : Payot. Le transfert, pp 408-425. Collection Petite Bibliothèque Payot
} 
concertation, l'écoute, autorisent la lecture des registres symboliques, affectifs, culturels que mobilisent les acteurs d'un espace pour en faire leur territoire.

\section{Vers une co-construction des politiques publiques sur les territoires}

L'enjeu est donc d'aborder les territoires à travers le prisme des représentations que s'en font leurs habitants. En effet, en considérant l'être-social-habitant comme acteur de sa territorialité, nous pouvons lire les productions territoriales qu'il engendre comme une représentation des stimuli produits par son environnement social. En d'autres termes, l'accent doit être mis sur une nouvelle lecture de l'interprétation que l'individu se fait de sa socialisation. Par conséquent, il ne s'agit plus de dégager des potentialités territoriales à grands renforts d'indicateurs quantitatifs, mais d'énoncer une réalité territoriale à un moment donné, c'est-à-dire celle vécue et ressentie par les habitants eux-mêmes. C'est un nouveau regard qui doit être privilégié permettant de traduire les informations que divulguent les personnes interrogées.

Ainsi, les politiques publiques de développement socio-territorial pourront résulter d'un consensus, d'un accord quant à ces modalités diverses de vivre l'espace. Les opérateurs du développement socio-territorial (services d'Etat, collectivités territoriales...) devront donc s'inscrire dans l'expérience démocratique. Faire émerger davantage de participation des individus dans le projet social et territorial global, en les interrogeant, en les aidant à construire ensemble un monde meilleur, en leur proposant des modalités nouvelles de démocratie participative, n'est-ce pas le projet qui transpire sous la notion de cohésion sociale ? Autant dire que poursuivre ce dessein dans le cadre des directions interministérielles de la cohésion sociale relève d'un vrai défi et appelle sans doute les agents à réinventer leurs pratiques professionnelles.

Nul doute que dans ce contexte, les expériences professionnelles, forgées sur le terrain au plus près des populations locales par les agents pourront servir de fondements afin que l'ingénierie sociale et territoriale ne soit pas seulement un vocable technocratique. Il s'agira alors pour les agents de construire, dans une dynamique de corporatisme pluriel, les valeurs, normes, algorithmes et images du référentiel cohésion sociale. S'ils accordent du crédit au fait que les individus convoquent leur "Arkhe-Pensée " pour vivre leur socialisation et leur territorialisation, alors les politiques publiques gagneront sans doute en efficacité.

Car, conduire des politiques publiques s'appuyant sur l'ingénierie sociale et territoriale impulserait de nouvelles dynamiques permettant :

De promouvoir la prise en compte des éléments qualitatifs dans les indicateurs des Budgets Opérationnels de Programmes (BOP) pour ne pas verser dans une culture du résultat et conserver le privilège professionnel de travailler non pas « pour» mais «avec» les habitants au risque que cette posture professionnelle soit chronophage,

- $\quad$ D’infléchir la promotion de politiques publiques qui prévoient l'entrée par l'animation de dispositifs privilégiant l'accompagnement individuel au bénéfice d'une approche collective plus globale fondée sur un projet social et territorial, 
- De favoriser les expériences originales locales fondées sur des diagnostics sociaux et territoriaux établis en concertation avec un large panel d'interlocuteurs dont les analyses vécues seraient largement entendues,

D’appréhender l'animation des politiques publiques dans une approche transversale où les logiques de transformations sociales seraient reconnues et valorisées au même titre que les logiques de réparations sociales.

A cet égard, c'est ce qu'appelait le Haut-Commissaire de la Jeunesse de ses vœux dans sa lettre du 10 décembre 2009 à l'attention des préfets de régions ayant pour objet les missions de l'Etat en matière de jeunesse, d'éducation populaire et de vie associative : "les missions de l'Etat en matière de jeunesse, d'éducation populaire et de vie associative doivent (y) trouver une nouvelle source de visibilité et d'efficacité pour les partenaires de l'Etat, les collectivités, les associations et l'ensemble des publics, notamment les jeunes ». Aux agents donc de bâtir ces nouvelles sources de visibilité et d'efficacité de leurs missions de service public. Quant à l'Etat, il lui incombe d'octroyer du temps aux agents pour qu'ils réalisent cette concertation-participation avec le plus grand nombre.

\section{Bibliographie :}

ASCHER F., 2004. Les nouveaux principes de l'urbanisme. La tour d'Aigues: Aube. Collection Poche Essai.

AUGE M., 2002. Non-lieux. Introduction à une anthropologie de la surmodernité. Paris : Seuil. Collection La Librairie du XXI siècle.

BOURDIEU P., 1993. La misère du monde. Paris : Seuil. Collection Points Essais.

DE CERTEAU M., 1990. L'invention du quotidien, I arts de faire. Paris: Gallimard. Collection Folio Essais.

DE RADKOWSKI GH., 2002. Anthropologie de l'habiter : Vers le nomadisme. Paris : PUF. DONZELOT J., 1999. « La nouvelle question urbaine », in Esprit.

DONZELOT J., 2004. «La ville à trois vitesses : relégation, périurbanisation, gentrification », in Esprit.

ELIADE M., [1965], 2002. Le sacré et le profane. Paris : Gallimard. Collection Folio Essais. FERRIER JP., 1998. Le contrat géographique ou l'habitation durable des territoires, Antée 2. Lausanne : Payot.

HEIDEGGER M., [1954] 2001. Essais et conférences. Paris : Gallimard. Collection Tel.

HELUWAERT M., 2002. «Les conditions d'apparition du ministère de la Jeunesse et des Sports » in Revue du comité d'histoire des ministères chargés de la jeunesse et des sports, 2537.

HOYAUX AF., 2006. «Pragmatique phénoménologique des constructions territoriales et idéologiques dans le discours des habitants », in L'espace géographique, 3, 271-285.

LABORIT H., 2000. La nouvelle grille. Paris : Gallimard. Collection Folio Essais.

LIPOVETSKY G, CHARLES S., 2004. Les temps hypermodernes. Paris : Grasset. Collection Nouveau collège de Philosophie.

MOLES A, ROHMER E., 2003. Psychosociologie de 1'espace. Paris : Harmattan. Collection villes et entreprises.

MONGIN O., 2005. La condition urbaine. La ville à l'heure de la mondialisation. Paris : Seuil. Collection La couleur des idées. 
MIGNON JM., 2007. Une histoire de l'éducation populaire. Paris : La découverte. Collection Alternatives Sociales.

MORIN E., 1991. La méthode, 4 - Les Idées, leur habitat, leur vie, leurs mœurs, leur organisation. Paris : Seuil. Collection Points Essais.

MORIN E., 2001. La méthode-Tome III, La connaissance de la connaissance. Anthropologie de la connaissance. Paris : Seuil. Collection Points Essais.

MULLER P., [1990], 2003. Les politiques publiques. Paris : PUF. Collection que sais-je ?

RIFKIN J., 2000. L'âge de l'accès: La révolution de la nouvelle économie. Paris : La Découverte. Collection Cahiers libres.

ROUX M., 1999. Géographie et complexité, Les espaces de la nostalgie. Paris : Harmattan. Collection L'Ouverture Philosophique.

SLOTERDIJK P., 2003 Bulles. Sphères I. Paris : Hachette. Collection Pluriel Philosophie.

Rapport 2009 de l'Observatoire National des Zones Urbaines Sensibles (ONZUS). Secrétariat Général du CIV, 270 p.

Rapport du groupe de travail sur l'ingénierie sociale, présidé par Annick Morel. 2009, 50 p.

Dossier de Presse des Etats Généraux des Sports Urbains. 16 Décembre 2009. Paris, Stade Pierre de Coubertin.

Décret n²009-1484 du 3 décembre 2009. Directions départementales interministérielles.

Décrets n²009-1540 du 10 décembre 2009. Directions Régionales de la Jeunesse, des Sports et de la Cohésion Sociale.

Circulaires du Premier Ministre du 19 septembre 2008.

Lettre de Martin Hirsch, Haut-Commissaire à la Jeunesse du 10 décembre 2009 ayant pour objet les missions de l'Etat en matière de jeunesse, d'éducation populaire et de vie associative 